\title{
Remarks on the McKay Conjecture
}

\author{
Geoffrey Mason
}

Received: 17 July 2008 / Accepted: 25 August 2008 / Published online: 17 February 2009

(C) The Author(s) 2009. This article is published with open access at Springerlink.com

\begin{abstract}
The McKay Conjecture (MC) asserts the existence of a bijection between the (inequivalent) complex irreducible representations of degree coprime to $p$ ( $p$ a prime) of a finite group $G$ and those of the subgroup $N$, the normalizer of Sylow $p$-subgroup. In this paper we observe that MC implies the existence of analogous bijections involving various pairs of algebras, including certain crossed products, and that $\mathrm{MC}$ is equivalent to the analogous statement for (twisted) quantum doubles. Using standard conjectures in orbifold conformal field theory, MC is equivalent to parallel statements about holomorphic orbifolds $V^{G}, V^{N}$. There is a uniform formulation of $\mathrm{MC}$ covering these different situations which involves quantum dimensions of objects in pairs of ribbon fusion categories.
\end{abstract}

Keywords McKay correspondence - Quantum double

Mathematics Subject Classification (2000) 20C05

\section{Introduction}

The following notation will be used throughout the paper: $G$ is a finite group, $p$ a prime, $P$ a Sylow $p$-subgroup of $G, N=N(P)$ the normalizer of $P$ in $G, G^{\prime}$ the commutator subgroup, $X$ a (finite, non-empty, left-) $G$-set, $\phi$ the Euler phi-function.

Presented by Jon Carlson.

Supported by grants from the NSF, NSA, and faculty research funds granted by the University of California at Santa Cruz.

G. Mason $(\varangle)$

Department of Mathematics,

University of California at Santa Cruz,

Santa Cruz, CA 95064, USA

e-mail: gem@cats.ucsc.edu 
All algebras and modules are finite-dimensional and defined over $\mathbb{C}$. $\mathbb{C}[G]$ is the group algebra of $G$ and $\mathbb{C}[G]^{*}$ the dual group algebra.

For an algebra $A$, let

$\mu(A)=\#$ inequivalent simple $A$-modules of dimension coprime to $p$.

We say that a pair of algebras $(A, B)$ is an $M$-pair in case $\mu(A)=\mu(B)$. The McKay Conjecture $(\mathrm{MC})$ is the assertion that $(\mathbb{C}[G], \mathbb{C}[N])$ is an M-pair. The reader may consult the paper [7] of Isaacs, Malle and Navarro for the current status of this conjecture. The idea of the present paper is to extend $\mathrm{MC}$ beyond its original formulation for groups. First we show how it may be extended to large classes of algebras that are not group algebras. Examples include crossed product algebras, where we show that $\left(\mathbb{C}[H]^{*} \#_{\sigma} \mathbb{C}[G], \mathbb{C}[H]^{*} \#_{\sigma} \mathbb{C}[N]\right)$ is an M-pair. Here, $G$ acts on the group $H$ and $\sigma$ is a certain 2-cocycle. (See $[8,11]$ for background.) A particularly interesting case is that of quantum doubles $D(G)$ (see $[4,9]$ and below for more details). In this case we establish

MC is true if, and only if, $(D(G), D(N))$ is an M-pair for all $G$ and $N$.

Note that quantum doubles $D(G)$ are generally not group algebras (unless $G$ is abelian).

For a multiplicative 3-cocycle $\omega \in Z^{3}\left(G, \mathbb{C}^{*}\right)$, we show that MC implies the same result for twisted quantum doubles. That is, $\left(D^{\omega}(G), D^{\omega}(N)\right)$ is an M-pair. Now there is a standard Ansatz in orbifold conformal field theory (CFT) due to Dijkgraaf et al. [6] which, when interpreted appropriately, says that the tensor category $D^{\omega}(G)$-Mod is equivalent to the module category $V^{G}$-Mod of a so-called holomorphic $G$-orbifold for a suitable vertex operator algebra $V$ admitting $G$ as automorphisms. Therefore, granted the DPR conjecture, MC is equivalent to a CFT-formulation involving a bijection between certain sets of simple modules for $V^{G}$ and $V^{N}$. It is not necessary for the reader to be familiar with this language; the point is simply that modules for $V^{G}$ are infinite-dimensional and the idea of an M-pair based on definition (1) makes no sense. In fact, all three types of M-pairs that we have discussed (i.e. for groups, (quasi-)Hopf algebras and orbifolds) may be uniformly described in the following setting: a pair of ribbon categories admitting a bijection between objects whose quantum dimension is integral and coprime to $p$.

All of the proofs in this paper are elementary and involve nothing beyond a few facts about finite groups, their representations, and their cohomology. In Section 2 we discuss some algebras $D_{X}(G)$ constructed from $G$ and a $G$-set $X$ and show that MC implies that $\left(D_{X}(G), D_{X}(N)\right.$ is an M-pair. We also establish Eq. 2. In Section 3 we carry out the twisted analog of this construction. Together, these results cover several of the connections with crossed products and twisted quantum doubles mentioned above. In Section 4 we discuss the connections with CFT and ribbon categories. We assure the reader that no knowledge of CFT is required to understand the contents of this paper.

We thank Siu-Hung $\mathrm{Ng}$, Robert Boltje and Gabriel Navarro for their interest and comments on earlier versions of this paper, and the referee for helpful remarks. 


\section{The Algebras $D_{X}(G)$}

We use the following additional notation: for $H \leq G, g \in G, H^{g}=\left\{g^{-1} h g \mid h \in H\right\}$. For $x \in X, \operatorname{Stab}_{G}(x)=\{g \in G \mid g \cdot x=x\}$.

We now introduce the algebras $D_{X}(G)$, which were mentioned briefly in [9]. Let $\mathbb{C}[X]^{*}$ be the space of complex-valued functions on $X$. One sees that it is a $G$-module algebra, as follows. The algebra structure is pointwise multiplication, with basis the Dirac delta functions

$$
e(x): y \mapsto \delta_{x, y} \quad x, y \in X
$$

Thus

$$
e(x) e(y)=\delta_{x, y} e(x) .
$$

$G$ acts on the left of $\mathbb{C}[X]^{*}$ as algebra automorphisms via

$$
g: e(x) \mapsto e(g \cdot x)
$$

Consider the linear space

$$
D_{X}(G)=\mathbb{C}[X]^{*} \otimes_{\mathbb{C}} \mathbb{C}[G] .
$$

It becomes an algebra via the product

$$
\begin{aligned}
(e(x) \otimes g)(e(y) \otimes h) & =e(x) e(g \cdot y) \otimes g h \\
& =\delta_{x, g \cdot y} e(x) \otimes g h
\end{aligned}
$$

for $x, y \in X$ and $g, h \in G$. One readily checks that this is associative. There is a decomposition into 2-sided ideals

$$
D_{X}(G)=\bigoplus_{Y} D_{Y}(G)
$$

where $Y$ ranges over the (transitive) $G$-orbits of $X$. For the most part, this reduces questions about $D_{X}(G)$ for general $X$ to the transitive case.

A special example of this construction is the quantum double of $G$. Here, we take $X=G_{\text {conj }}$, i.e. $X=G$ and the left action of $G$ is left conjugation $g: x \mapsto g x g^{-1}$. In this case we write $D(G)$ in place of $D_{G_{\text {conj }}}(G) . D(G)$ is in fact a Hopf algebra, but at the moment we only require the algebra structure.

Next we describe the category of (left-) $D_{X}(G)$-modules (cf. [6, 8, 9]). For $x \in X$ and a left $\operatorname{Stab}_{G}(x)$-module $V$, set $V_{x}=e(x) \otimes V$. This is a left $\mathbb{C}[X]^{*} \otimes \operatorname{Stab}_{G}(x)$ module via

$$
(e(y) \otimes g) .(e(x) \otimes v)=e(y) e(x) \otimes g . v=\delta_{x, y} e(x) \otimes g . v, g \in \operatorname{Stab}_{G}(x) .
$$

From Eq. 4 it follows that $\mathbb{C}[X]^{*} \otimes \operatorname{Stab}_{G}(x)$ is a subalgebra of $D_{X}(G)$, so that $D_{X}(G) \otimes \mathbb{C}[X]^{*} \otimes \operatorname{Stab}_{G}(x)=V_{x}$ is a left $D_{X}(G)$-module.

Proposition 2.1 Suppose that $X$ is a transitive G-set and $x \in X$. Then the map

$$
\begin{aligned}
\mathbb{C}\left[\operatorname{Stab}_{G}(x)\right]-\operatorname{Mod} & \rightarrow D_{X}(G)-\operatorname{Mod}, \\
V & \mapsto D_{X}(G) \otimes_{\mathbb{C}[X]^{*} \otimes \operatorname{Stab}_{G}(x)} V_{x},
\end{aligned}
$$

is a Morita equivalence. 
Proof For this and more, see [9] and Section 3 of [8]. In these references $X$ is a group, but this is not necessary and the proofs go through without change. Via the natural identification $\mathbb{C}\left[\operatorname{Stab}_{G}(x)\right] \stackrel{\cong}{\rightarrow} e(x) \otimes \mathbb{C}\left[\operatorname{Stab}_{G}(x)\right], g \mapsto e(x) \otimes g$, the object map inverse to $(7)$ is $W \mapsto(e(x) \otimes 1) W$.

Proposition 2.2 Suppose that $X$ is a transitive $G$-set and $x \in X$. The following hold:

(a) If $p$ does not divide $|X|$ then $\left(D_{X}(G), \operatorname{Stab}_{G}(x)\right)$ is an M-pair.

(b) If $p$ divides $|X|$ then $\mu\left(D_{X}(G)\right)=0$.

Proof Let $T$ be a set of right coset representatives in $G$ for $\operatorname{Stab}_{G}(x)$, so that there is a disjoint union $G=\cup_{t \in T} t \operatorname{Stab}_{G}(x)$. Because $X$ is transitive then $X=\{t . x \mid t \in T\}$. From Eq. 4, observe that $D_{X}(G)$ is a free right $\mathbb{C}[X]^{*} \otimes \operatorname{Stab}_{G}(x)$-module with free basis $\{e(t . x) \otimes t \mid t \in T\}$. Using this observation, it follows that for a left $\operatorname{Stab}_{G}(x)$ module $V$,

$$
\begin{aligned}
\operatorname{dim}\left(D_{X}(G) \otimes_{\mathbb{C}[X]^{*} \otimes \operatorname{Stab}_{G}(x)} V_{x}\right) & =|T| \operatorname{dim} V \\
& =|X| \operatorname{dim} V .
\end{aligned}
$$

Therefore, in the Morita equivalence (7) modules of dimension $d$ are mapped to modules of dimension $d|X|$. Parts (a) and (b) both follow immediately from this.

Lemma 2.3 $\mu\left(D_{X}(G)\right)=\sum_{Y} \mu\left(D_{Y}(G)\right)$ where $Y$ ranges over the G-orbits of $X$.

Proof This follows from the decomposition (5) into 2-sided ideals.

We will also need the following standard result.

Lemma 2.4 The number of G-orbits of $X$ of cardinality coprime to $p$ is equal to the number of $N$-orbits of $X$ of cardinality coprime to $p$.

Proof By considering the decomposition of $X$ into $G$-orbits, we see that we must prove the following assertion:

If $X$ is a transitive $G$-set, then either (a) $p$ divides $|X|$ and $N$ has no orbits of cardinality coprime to $p$, or (b) $p$ does not divide $|X|$ and there is a unique $N$-orbit of cardinality coprime to $p$.

Assume, then, that $X$ is a transitive $G$-set, and that $x, y \in X$ lie in $N$-orbits of cardinality coprime to $p$. In such an $N$-orbit, $P$ must fix at least one, and therefore all, elements in the $N$-orbit. In particular, $P$ lies in the stabilizers of both $x$ and $y$. By transitivity there is $g \in G$ with $g . x=y$. Then $P$ and $P^{g}$ are both Sylow $p$-subgroups of $\operatorname{Stab}_{G}(x)$ and by Sylow's theorem there is $t \in \operatorname{Stab}_{G}(x)$ such that $P^{g t}=P$. Then $g t \in N$ and $(g t) . x=y$. This shows that $x, y$ lie in the same $N$-orbit, so that there is at most one $N$-orbit of cardinality coprime to $p$. Equation (8) is easily deduced from this, and the lemma is proved. 
Consider the following statements:

MC1 : $(\mathbb{C}[G], \mathbb{C}[N])$ is an M-pair for all $G$,

MCD : $(D(G), D(N))$ is an M-pair for all $G$,

MCX : $\left(D_{X}(G), D_{X}(N)\right)$ is an M-pair for all $G$ and all $X$,

MCT : $\left(D_{X}(G), D_{X}(N)\right)$ is an M-pair for all $G$ and all transitive $X$.

The McKay Conjecture is of course the assertion that MC1 is true.

Theorem 2.5 MC1, MCD, MCX and MCT are equivalent statements.

Proof

$M C X \Leftrightarrow M C T \quad$ This follows from Lemma 2.4.

$M C T \Rightarrow M C 1 \quad$ This holds because if $X=\mathbf{1}$ is the one-element set, then $D_{X}(G)=$ $\mathbb{C}[G]$.

$M C 1 \Rightarrow M C T \quad$ Let $X$ be a transitive $G$-set. If $p$ divides $|X|$ then $\mu\left(D_{X}(G)\right)=0$ by Proposition 2.2(b). Similarly, $\mu\left(D_{X}(N)\right)=0$ by Lemma 2.3, (8)(a), and Proposition 2.2(b) (applied to $N$ ). Now suppose that $p$ does not divide $|X|$. Then $\mu\left(D_{X}(G)\right)=\mu\left(\mathbb{C}\left[\operatorname{Stab}_{G}(x)\right]\right)$ for any $x \in X$ by Proposition 2.2(a). Moreover by Lemma 2.4 there is a unique $N$-orbit of cardinality coprime to $p$, call it $Y \subseteq X$. By Lemmas 2.3, (8) and Proposition 2.2 once more we find that $\mu\left(D_{X}(N)\right)=\mu\left(D_{Y}(N)\right)=\mu\left(\mathbb{C}\left[\operatorname{Stab}_{N}(y)\right]\right)$ for $y \in Y$. Note that because $|Y|$ is coprime to $p$ then $P \leq \operatorname{Stab}_{G}(y)$ and $N_{\operatorname{Stab}_{G}(y)}(P)=\operatorname{Stab}_{N}(y)$. The assumption that $M C 1$ holds (applied to $\left.\operatorname{Stab}_{G}(y)\right)$ tells us that $\mu\left(\mathbb{C}\left[\operatorname{Stab}_{G}(y)\right]\right)=\mu\left(\mathbb{C}\left[\operatorname{Stab}_{N}(y)\right]\right)$, whence $\mu\left(D_{X}(G)\right)=\mu\left(D_{X}(N)\right)$.

$M C X \Rightarrow M C D$ Let $Y_{1}, \ldots, Y_{h}$ be the $N$-orbits of $G_{\text {conj }}$ of cardinality coprime to $p$. We have $Y_{i} \subseteq N$ for each index $i$, so that they are also the $N$-orbits of $N_{\text {conj }}$ of cardinality coprime to $p$. Taking $X=G_{\text {conj }}$, MCX together with Lemma 2.3 and Proposition 2.2, we conclude that $\mu(D(G))=\mu\left(D_{G_{\text {conj }}}(N)\right)=\sum_{i=1}^{h} \mu\left(D_{Y_{i}}(N)\right)=$ $\mu\left(D_{N_{\text {conj }}}(N)\right)=\mu(D(N))$, as required.

$M C D \Rightarrow M C 1$ We prove this using induction on $|G|$. Retain the notation of the last paragraph, and choose $y_{i} \in Y_{i}$. By Lemma 2.4, $y_{1}, \ldots, y_{h}$ are representatives for the $G$-orbits of $G_{\text {conj }}$ (i.e., conjugacy classes of $G$ ) of cardinality coprime to $p$. By Proposition 2.2, $\mu(D(G))=\sum_{i=1}^{h} \mu\left(\mathbb{C}\left[C_{G}\left(y_{i}\right)\right]\right)$. Since each $y_{i} \in N$, we similarly have $\mu(D(N))=\sum_{i=1}^{h} \mu\left(\mathbb{C}\left[C_{N}\left(y_{i}\right)\right]\right)$. If $C_{G}\left(y_{i}\right)$ is a proper subgroup of $G$ then by induction $\mu\left(\mathbb{C}\left[C_{G}\left(y_{i}\right)\right]\right)=\mu\left(\mathbb{C}\left[C_{N}\left(y_{i}\right)\right]\right)$. Then the assumption $M C D$ tells us that $\sum_{i^{\prime}} \mu(\mathbb{C}[G])=\sum_{i^{\prime}} \mu(\mathbb{C}[N])$ where $i^{\prime}$ ranges over those indices for which $y_{i^{\prime}}$ lies in the center $Z(G)$ of $G$. We conclude that $|Z(G)| \mu(\mathbb{C}[G])=|Z(G)| \mu(\mathbb{C}[N])$, whence $\mu(\mathbb{C}[G])=\mu(\mathbb{C}[N])$. This completes the proof of the theorem. 


\section{Twisted Algebras}

In this section we explain how to extend the results of the previous section to the twisted case, i.e. the incorporation of a cocycle. Let $\theta \in Z^{2}\left(G, \mathbb{C}^{*}\right)$ be a (normalized) multiplicative 2-cocycle. Thus $\theta: G^{2} \rightarrow \mathbb{C}^{*}$ satisfies the identities

$$
\begin{aligned}
\theta(h, k) \theta(g, h k) & =\theta(g h, k) \theta(g, h), \quad g, h, k \in G, \\
\theta(1, g) & =\theta(g, 1)=1 .
\end{aligned}
$$

The corresponding twisted group algebra is $\mathbb{C}^{\theta}[G]$. It has the same underlying linear space as $\mathbb{C}[G]$ with multiplication $g \circ h=\theta(g, h) g h$ for $g, h \in G$. The cocycle identities ensure that this is an associative algebra with identity element 1 . For a subgroup $H \leq G$ we identify $\theta$ with its restriction $\operatorname{Res}_{H}^{G} \theta$ to $H$. Then $\mathbb{C}^{\theta}[H]$ is a subalgebra of $\mathbb{C}^{\theta}[G]$. For more information on this subject, including results that we use below, see for example [3].

The cohomological analog of Proposition 2.2(b) is the following

Lemma 3.1 Suppose that the cohomology class $[\theta] \in H^{2}\left(G, \mathbb{C}^{*}\right)$ determined by $\theta$ has order $k$. If $k$ is divisible by $p$ then $\mu\left(\mathbb{C}^{\theta}[G]\right)=\mu\left(\mathbb{C}^{\theta}[N]\right)=0$.

Proof One knows (loc.cit.) that there is a central extension

$$
1 \rightarrow Z \rightarrow L \stackrel{\pi}{\rightarrow} G \rightarrow 1
$$

such that $\mathbb{Z}_{k} \cong Z \leq L^{\prime}$, and $\mathbb{C}^{\theta}[G]$ is the algebra summand of $\mathbb{C}[L]$ corresponding to the irreducible representations of $L$ in which a generator $z$ of $Z$ acts as multiplication by a prescribed primitive $k$ th root of unity, say $\lambda$. If $V$ is a simple $\mathbb{C}^{\theta}[G]$-module of dimension $d$ then the determinant of $z$ considered as operator on $V$ is clearly $\lambda^{d}$. On the other hand $z \in L^{\prime}$, so that this determinant is necessarily 1 . So $\lambda^{d}=1$, whence $k \mid d$. In particular, if $p \mid k$ then $\mu\left(\mathbb{C}^{\theta}[G]\right)=0$.

Now it is well-known that the restriction map $\operatorname{Res}_{N}^{G}: H^{2}\left(G, \mathbb{C}^{*}\right) \rightarrow H^{2}\left(N, \mathbb{C}^{*}\right)$ is an injection on the $p$-part of $H^{2}\left(G, \mathbb{C}^{*}\right)$. In particular, if $p \mid k$ then $\operatorname{Res}_{N}^{G}[\theta]$ is divisible by $p$. Then the result of the last paragraph also applies to $\mathbb{C}^{\theta}[N]$, and we obtain $\mu\left(\mathbb{C}^{\theta}[N]\right)=0$. This completes the proof of the lemma.

The McKay Conjecture implies that the twisted analog is also true. This is the content of

Proposition 3.2 Suppose that MC1 holds. Then $\left.\left(\mathbb{C}^{\theta}[G]\right), \mathbb{C}^{\theta}[N]\right)$ is an M-pair for all $G$ and all $\theta$.

Proof Let the notation be as in Lemma 3.1. Although it is not really necessary to do so, because of Lemma 3.1 we may, and shall, assume that $k$ is not divisible by $p$. Let $P_{1}$ be a Sylow $p$-subgroup of $L$ with $\pi: P_{1} \cong P$. Applying MC1 to pairs $\left(L / Z_{0}, N_{L}\left(P_{1}\right) / Z_{0}\right)$ with $Z_{0} \leq Z$, we see that the number $l$ of irreducible representations of both $\mathbb{C}[L]$ and $\mathbb{C}\left[N_{L}\left(P_{1}\right)\right]$ which have degree coprime to $p$ and in which $z$ acts as some primitive $k$ th root of unity are equal. Since $\mathbb{C}^{\theta}[G]$ is the algebra 
summand of $\mathbb{C}[L]$ corresponding to $\lambda$, then $\mu\left(\mathbb{C}^{\theta}[G]\right)=l / \phi(k)$. On the other hand, $N_{L}\left(P_{1}\right)=Z K$ where $K \leq L$ is such that $\mathbb{C}^{\theta}[N]$ is the algebra summand of $\mathbb{C}[K]$ corresponding to $\lambda^{t}$, where $t \mid k$ and $k / t$ is the order of $\operatorname{Res}_{N}^{G}[\theta]$. By slightly modifying the previous argument, we also find that $\mu\left(\mathbb{C}^{\theta}[N]\right)=l / \phi(k)$, and the Proposition is proved.

We can now treat the twisted version of $D_{X}(G)$. Let $U=U\left(\mathbb{C}[X]^{*}\right)$ be the group of units in $\mathbb{C}[X]^{*}$. Then

$$
U=\left\{\sum \lambda_{x} e(x) \mid \lambda_{x} \neq 0\right\}
$$

is a multiplicative left $G$-module. Let $\alpha \in Z^{2}(G, U)$ be a normalized 2-cocycle with coefficients in $U$, and set $\alpha(g, h)=\sum_{x \in X} \alpha_{x}(g, h) e(x)$. Here, the cocycle property amounts to the identity

$$
\alpha_{x}(g, h) \alpha_{x}(g h, k)=\alpha_{x}(g, h k) \alpha_{g^{-1} . x}(h, k) .
$$

Define $D_{X}^{\alpha}(G)$ to be the linear space $D_{X}(G)$ with multiplication being the twisted version of Eq. 4. That is,

$$
(e(x) \otimes g)(e(y) \otimes h)=\alpha_{x}(g, h) \delta_{x, g . y} e(x) \otimes g h .
$$

Equation 9 is exactly what is needed to show that Eq. 10 is associative. Note also from Eq. 9 that for fixed $x \in X, \alpha_{x}$ defines an element in $Z^{2}\left(\operatorname{Stab}_{G}(x), \mathbb{C}^{*}\right)$ and that as a subalgebra of $D_{X}^{\alpha}(G), e(x) \otimes \mathbb{C}\left[\operatorname{Stab}_{G}(x)\right] \cong \mathbb{C}^{\alpha_{x}}\left[\operatorname{Stab}_{G}(x)\right]$. The proof of Proposition 2.1 still applies in this situation (cf. [8]). It provides a Morita equivalence of categories

$$
\mathbb{C}^{\alpha_{x}}\left[\operatorname{Stab}_{G}(x)\right]-\operatorname{Mod} \stackrel{\sim}{\rightarrow} D_{X}^{\alpha}(G) \text {-Mod. }
$$

The proof of the twisted version of Theorem 2.5 then goes through too. We just state a part of this as

Theorem 3.3 Suppose that MC1 holds. Then $\left(D_{X}^{\alpha}(G),\left(D_{X}^{\alpha}(N)\right)\right.$ is an M-pair for all $G, X$ and $\alpha$.

Special cases of $D_{X}^{\alpha}(G)$ include certain kinds of crossed products and abelian extensions of Hopf algebras. See, for example, [8] for further details.

Once again the case of the quantum double, when $X=G_{\text {conj }}$, is of special interest (cf. $[2,5,6,9]$ for more details and further background.) Here, one twists $D(G)$ by a normalized three cocycle $\omega \in Z^{3}\left(G, \mathbb{C}^{*}\right)$. The resulting object is denoted by $D^{\omega}(G)$. It is a quasi-Hopf algebra, but not a Hopf algebra in general. To connect with previous paragraphs, we observe that there is a map [6]

$$
Z^{3}\left(G, \mathbb{C}^{*}\right) \rightarrow Z^{2}\left(G, G_{\text {conj }}\right)
$$

for which

$$
\alpha_{x}(g, h)=\frac{\omega(x, g, h) \omega\left(g, h,(g h)^{-1} x(g h)\right)}{\omega\left(g, g^{-1} x g, h\right)} .
$$


There is a natural interpretation of this map in terms of the loop space $L B G$, but we will not need it. The twisted product in $D^{\omega}(G)$ is as in Eq. 10 using Eq. 11. This gives the algebra structure, and as before leads to

Theorem 3.4 Suppose that MC1 holds. Then $\left(D^{\omega}(G), D^{\omega}(N)\right)$ is an M-pair for all $G$ and $\omega$.

The statement and proof of Theorem 3.4 only require the algebra structure of $D^{\omega}(G)$. However, we will make use of other structural features of $D^{\omega}(G)$ in the next section.

\section{Orbifolds and Ribbon Categories}

We refer the interested reader to [5] for background concerning vertex operator algebras. Let $V$ be a holomorphic vertex operator algebra admitting $G$ as a group of automorphisms, with $V^{G}$ the subalgebra of $G$-invariants. One expects that the module category $V^{G}$-Mod is a (braided, ribbon) tensor category and that it equivalent to the tensor category $D^{\omega}(G)$-Mod for a 3-cocycle $\omega$ which describes the associativity constraint in $V^{G}$-Mod. If this is so, we deduce from Theorem 3.4 that there are bijections between the simple objects of $V^{G}$-Mod and $V^{N}$-Mod which themselves correspond to the simple modules of $D^{\omega}(G)$-Mod and $D^{\omega}(N)$-Mod respectively which have dimension coprime to $p$.

We seek a direct definition of an M-pair for modules over orbifolds such as $V^{G}$ and $V^{N}$. We cannot use Eq. 1 as it stands because it makes no sense for infinitedimensional spaces such as a module over a vertex operator algebra. Instead, we can make use of the expected structure of $V^{G}$-Mod as a ribbon tensor category, whereby the objects have a quantum dimension. Indeed, $D^{\omega}(G)$-Mod has a canonical ribbon structure (cf. $[1,10]$ ), and the quantum dimensions of simple objects are the usual dimensions. Granted the equivalence of $V^{G}$-Mod and $D^{\omega}(G)$-Mod, it follows that the quantum dimension of simple objects in $V^{G}$-Mod are also integers. Then the definition of an M-pair makes sense if we use quantum dimension in place of dimension.

Thus we arrive at the following situation: a pair of ribbon fusion categories $\mathcal{G}, \mathcal{N}$ whose simple objects have quantum dimensions that are rational integers. We say that $(\mathcal{G}, \mathcal{N})$ is an M-pair if $\mu(\mathcal{G})=\mu(\mathcal{N})$, where we use (1) with quantum dimension in place of dimension in order to define $\mu$. As we have seen, taking $\mathcal{G}$ to be $\mathbb{C}[G]$ Mod, $D^{\omega}(G)$-Mod or $V^{G}$-Mod and $\mathcal{N}$ to be $\mathbb{C}[N]$-Mod, $D^{\omega}(N)$-Mod or $V^{N}$-Mod respectively results (conjecturally) in an M-pair. Furthermore, the three versions of $\mathrm{MC}$ for groups, quantum doubles of groups, and holomorphic orbifolds, are equivalent.

Open Access This article is distributed under the terms of the Creative Commons Attribution Noncommercial License which permits any noncommercial use, distribution, and reproduction in any medium, provided the original author(s) and source are credited. 


\section{References}

1. Altschuler, D., Coste, A.: Quasi-quantum groups, knots, three-manifolds, and topological field theory. Comm. Math. Phys. 150(1), 83-107 (1992)

2. Kassel, C.: Quantum Groups, Graduate Texts in Mathematics, vol. 155. Springer, New York (1995)

3. Curtis, C., Reiner, I.: Methods of Representation Theory, vol. 1. Wiley-Interscience, New York (1981)

4. Drinfeld, V.: Quasi-Hopf algebras. Leningr. Math. J. 1, 1419-1457 (1990)

5. Dong, C., Mason, G.: Vertex operator algebras and moonshine: a survey. In: Adv. Studies in Pure Math. vol. 24, pp. 101-136. Progress in Algebraic Combinatorics, Math. Soc. of Japan, Tokyo (1996)

6. Dijkgraaf, R., Pasquier, V., Roche, P.: Quasi-quantum groups related to orbifold models. In: Modern Quantum Field Theory (Bombay, 1990), pp. 375-383. World Scientific, Singapore (1991)

7. Isaacs, M., Malle, G., Navarro, G.: A reduction theorem for the McKay conjecture. Invent. Math. 170, 33-101 (2007)

8. Kashina, Y., Mason, G., Montgomery, S.: Computing the Frobenius-Schur indicator for Abelian extensions of Hopf algebras. J. Algebra 251, 888-913 (2002)

9. Mason, G.: The quantum double of a finite group and its role in conformal field theory. In: Groups '93 Galway/St. Andrews, Lond. Math. Soc. Lect. Notes Ser. 212, vol. 2. CUP (1995)

10. Mason, G., Ng, S.-H.: Central invariants and Frobenius-Schur indicators for semi-simple quasiHopf algebras. Adv. Math. 190, 161-195 (2005)

11. Montgomery, S.: Hopf Algebras and Their Actions on Rings, CBMS Number 82. Amer. Math. Soc. (1993) 